\title{
ORIGINAL CONTRIBUTION Analysis of Questionnaire Surveys Conducted in Higher Education Institutions
from the Perspective of the Institutional Research for Teaching and Learning
}

\author{
Yoshioka Tsuyoshi ${ }^{1 *}$, Morikura Yusuke ${ }^{2}$, Kobayashi Ryo ${ }^{3}$, Teruya Kensaku ${ }^{4}$, Izumi Toshiro ${ }^{5}$ \\ 1,2,3,4,5 Teikyo Heisei University, Tokyo, Japan
}

\begin{abstract}
This study proposes a simplified Customer Satisfaction (CS) analysis procedure by summarizing the CS analysis method, which can be practically applied to questionnaire analysis from the perspective of Institutional Research for Teaching and Learning (IRTL). Most of the questionnaire surveys conducted in higher education institutions are mere aggregations of the percentage of responses. A course evaluation questionnaire assessment method applying CS analysis, which is often used in marketing, has already been established to solve this problem. However, this method cannot be practically implemented if IRTL organizations are not fully functional. Using this proposed method, it is possible to specifically analyze the items that need to be focused on and improved to enhance student satisfaction. Furthermore, this study also examines whether the proposed method could be applied to the analysis of questionnaires for student surveys that have been conducted in recent years. As a result, the proposed method based on the perspective of IRTL has also succeeded in summarizing the guidelines for higher education institutions to conduct survey analysis.
\end{abstract}

Index Terms - Higher Education, Questionnaire Analysis, Customer Satisfaction Analysis, IRTL, Faculty Development

Received: 21 January 2021; Accepted: 29 May 2021; Published: 24 July 2021

\section{Introduction}

In recent years, Information and Communication Technology (ICT) has made it easier to conduct surveys and compile questionnaires. The number of surveys conducted using questionnaires in higher education institutions has been increasing. For example, in Japanese universities, surveys for course evaluation by students were conducted individually by faculty members or some private universities until the early 1990s (Kurita, 2006). In the academic year 2018, 756 (approximately 99\%) out of 761 universities that responded to the survey for course evaluation implemented the questionnaires, and 729 (approximately 96\%) did so in all departments (Ministry of Education, Culture, Sports, Science and Technology (MEXT), 2020a). Almost all universities have implemented course evaluation questionnaires in recent years, and many universities have conducted questionnaires for student surveys (The University of Tokyo, n.d.; Waseda University, 2020). Large-scale student surveys have also been conducted in the United Kingdom and the United States. Following this, a nationwide student survey was planned in Japan, and a trial implementation was completed in 2019 (Ministry of Education, Culture, Sports, Science and Technology (MEXT), 2019). Furthermore, in the academic year 2020, because of the COVID-19 pandemic, it became necessary to conduct classes online. Questionnaire surveys on the implementation of online classes were conducted in many higher education institutions (Tohoku University, n.d.; Waseda University, 2020).

Conducting questionnaire surveys is very important to understand students' actual situations and environments. However, the more questionnaires there are, the more similar the questionnaire items will be, resulting in unnecessary costs. Even if a large amount of money and time is spent gathering and collecting a vast amount of information, conducting questionnaire surveys becomes futile if the information is not fully utilized. Therefore, if a survey is to be conducted, it is essential to understand the actual situation and conduct a thorough analysis to improve the situation and environment. However, at present, questionnaires in higher education institutions are analyzed only by tabulating and summarizing the percentage of responses. Although they can grasp the actual situation, they do not conduct an analysis that leads to improvement. This study discusses the questionnaires conducted in higher education institutions from the perspective of IRTL. The IRTL is defined as "the practice and research collecting, analyzing, and reporting data to improve teaching and learning" (Matsuda \& Watanabe, 2017). In this way, the IRTL mainly deals with data related to teaching and learning and focuses on improving education (Okada \& Torii, 2018). In other words, the questionnaire survey that the IRTL should aim to conduct does not merely focus on measuring the "satisfaction of students" and understanding the situation. It is based on the premise that clarifying educational issues can lead to education improvement and quality assurance. Hence, studies have been reported on analytical methods, such as customer satisfaction analysis (Endo, 2008; Matsumoto \& Tsukamoto, 2004; Minami, 2007, 2008; Minami \& Nakanishi, 2010) and text mining (Fushikida et al., 2012; Kamaga, 2015), which can be used to improve education and

\footnotetext{
Email: t.yoshioka@thu.ac.jp
} 
learning. However, because of the complexity of these methods, even if the analysis methods were established as research, it is difficult to improve teaching and learning by analyzing all the questionnaires unless the higher education institution has a well-functioning IRTL organization and is actively engaged in Faculty Development (FD) activities.

This study investigates why, in practice, it is difficult to utilize course evaluation questionnaire assessment using CS analysis from the perspective of IRTL. This paper discusses how to produce useful analytical results using practical CS analysis and improve the learning environment in higher education institutes. Furthermore, we mention questionnaires for student surveys and discuss how questionnaire surveys conducted in higher education institutions should be practically analyzed from the perspective of IRTL.

\section{Literature Review}

Students' course evaluations were introduced in the late 1960s (The University of Chicago, n.d.). In recent years, almost all universities in Japan have been conducting course evaluation questionnaires. However, 49.3\% (Ministry of Education, Culture, Sports, Science and Technology (MEXT), 2014) of the universities in the academic year 2012 and 67.9\% (Ministry of Education, Culture, Sports, Science and Technology (MEXT), 2020b) in the academic year 2018 indicated the following as a distinctive approach to course evaluation: "we provide opportunities to organizationally survey the results of class questionnaires and reflect them in class content, etc." Even though several universities leverage course evaluations by students to improve their teaching, they are not analyzed useful, and many universities only count the percentage of responses. Additionally, even universities that indicated the above-quoted answer often only return the aggregated results and the weighted mean of each item to the instructor. Incidentally, in the aggregation of the weighted mean of each item, such as the five-point scale for each item, weighted means are simply calculated without rationale by assigning a grade from one to five. A grade of "five" does not represent five times the grade of "one"; therefore, if two students provide grades of one and five, respectively, and the mean of the two grades is three, it cannot be said that this grade equals a grade provided by a student who provided a grade of three. Hence, it is futile to find a weighted mean (Endo, 2008). One can only understand whether the ratings are high or low by comparing them with the mean of the faculty or department, and their analysis is not useful. In other words, in many universities, the results of student course evaluation questionnaires are aggregated by the percentage of responses. Whereas in some universities, it is possible to compare the mean of each faculty or department with the weighted mean of one's course evaluation; however, there are few cases in which the results of course evaluation questionnaires are systematically analyzed in detail to improve the courses (Matsumoto \& Tsukamoto, 2004). Overall, course evaluation is not clearly linked to appropriate course improvement activities.

Various organizational efforts to use course evaluation effectively are also being considered and implemented. For instance, operations are integrated with the preparation of course improvement plans (Takahashi et al., 2005), a commendation system for instructors with high weighted mean values of course evaluations, and countermeasures for instructors with a low weighted mean (Minami \& Nakanishi, 2010). However, even in cases where the management is integrated with the preparation of a course improvement plan since a thorough analysis is not conducted, in the aggregated results (such as the weighted mean value of each item), instructors simply "reflect" on the low rating items and state their determination to "improve next time" (Endo, 2008). The commendation system for instructors with high mean scores in course evaluation and the countermeasures for instructors with low mean scores constitute an indirect or "external pressure" from instructors who receive feedback on their course evaluations (Minami \& Nakanishi, 2010). It is up to each instructor how to utilize the results of course evaluations, which are effectively provided as feedback to each instructor, and it cannot be said that this is a direct approach to the first step of utilizing course evaluations for course improvement (Minami \& Nakanishi, 2010). In the first place, the purpose of course evaluation is not to compare and compete with other instructors for superiority but to improve each course subject. It is dangerous to assume that by comparing the weighted mean values of each item, it is possible to compare their performance with that of other instructors (Endo, 2008). In such questionnaires, the results are greatly influenced by the subjectivity of the respondents, and it should be noted that it is difficult to draw conclusions that will lead to course improvement by comparing the weighted mean values by each item (Abe et al., 2006). The results of course evaluations are the real voices of the students, and they are valuable data for course improvement. Even if some problems exist, such as the lack of seriousness in the attitude of the students or their unfamiliarity with the evaluation process, the data truly reflect the nature of the students as they are. It also helps in identifying the gap between instructors and the students; the instructors who receive the feedback should not turn away from this reality (Matsumoto \& Tsukamoto, 2004).

Although conventional course evaluation questionnaires are discussed from a critical perspective, conducting the questionnaires and compiling the responses are understandably very important. However, even though course evaluations are conducted for the objective of improving courses, the reality is that the results are returned to the instructors, focusing only on the percentage of responses and the weighted mean, and efforts to improve the courses are entrusted to each instructor (Minami, 2007). It is an inadequate way to handle information that has been collected with a great deal of expense and time (Kawakami \& Yoshizumi, 2008); thus, it cannot be said that course evaluation is clearly linked to course improvement activities. The problem is that there are no clear guidelines on "how to link the results of the questionnaires to course improvement" (Endo, 2008).

\section{Methodology}

Analysis method of course evaluation questionnaires using CS analysis

The pertinent question is how the numerical data obtained from course evaluations can improve each course subject. One must realize that when the course evaluations are returned to instructors, not merely are the weighted mean values presented, but also suggestions for important improvements (or effective improvements) should be given as feedback. Based on this, it will be easier for instructors to make clear improvements at their level, even if there is no organized activity to improve each higher education institution; this will naturally lead to improvements in the course (Minami, 2007). Therefore, CS analysis can be considered a suitable method to solve this problem. CS analysis is one of the methods used mainly in the field of marketing. It is a general term for analysis methods used to develop products and management strategies that reflect customers' needs and does not refer to any specific analysis method (Matsumoto \& Tsukamoto, 2004). Essentially, CS analysis refers to a method of surveying customer satisfaction through questionnaires and analyzing the results. The purpose of this analysis method is to increase customer satisfaction while feeding back the analysis results to product development and management strategies (Matsumoto \& Tsukamoto, 2004). It is not efficient to allocate resources equally to all questions asked in the 
questionnaire for increasing customer satisfaction. Thus, it is important to derive a policy that will determine which items focus on efforts in terms of importance and satisfaction to increase customer satisfaction effectively (Minami, 2007).

This CS analysis will provide feedback on the index of need for improvement, showing which items need to be focused on for improvement; therefore, each instructor will find their guidelines to improve their courses (Minami \& Nakanishi, 2010). Students are not customers, and courses are not products; therefore, there may be some discomfort linking course evaluation to CS analysis. While satisfaction is not the only index of a good course (Endo, 2008), increasing student satisfaction with the course is one of the goals for course improvement. Research has been conducted to analyze course evaluation questionnaires using CS analysis (Endo, 2008; Matsumoto \& Tsukamoto, 2004; Minami, 2007). Furthermore, it was found that the level of the need for improvement in the course evaluation items related to appropriate teaching methods decreased as the overall satisfaction level increased, indicating that CS analysis has a certain validity (Minami, 2008).

CS analysis of course evaluation questionnaires from the perspective of IRTL

In the previous subsection, the application of CS analysis to course evaluation questionnaires was summarized. However, although these methods have been established as research, they are not suitable for applying to actual course evaluations and providing feedback to each instructor for course improvement. Therefore, only a few higher education institutions would regularly conduct the CS analysis of course evaluation questionnaires on a university-wide basis. This is because, in Japan, the course evaluation questionnaire is one of the areas covered by the IRTL. From the perspective of IRTL, analyzing the results of course evaluation questionnaires actually to lead to improvements in education is a prerequisite. In other words, even if CS analysis were used to analyze the results of course evaluation questionnaires, each instructor would need to have some knowledge of statistical methods to understand the results when they receive them; therefore, the results would not be meaningful from the perspective of IRTL unless the institution is a higher education institution with active FD activities and sufficient training. In addition, the more complex the analysis method, the more practical the problem of securing the resources for the IRTL staff to conduct the analysis. Incidentally, in North America, the Association for Institutional Research, a professional association of specialists in IR, was established in 1965 (Yamada, 2016). While there have already been more than half a century of accumulated practice and research in North America, it is still early days for the IRTL efforts in Japan. It needs to overcome some issues to function fully (Okada \& Torii, 2018). As mentioned above, although a method for analyzing course evaluation questionnaires using the CS analysis has been established, from the perspective of IRTL, there is the issue of "whether each instructor has the statistical knowledge to understand the results of the analysis", as well as the issue of "whether each university has the resources to use the method for analysis" in the first place. Therefore, from the perspective of IRTL, this study proposes a CS analysis of course evaluation questionnaires at a level that is practically feasible for each higher education institution to undertake.

First, the purpose of the course evaluation questionnaire is to increase the overall satisfaction of each individual class. In other words, in this study, a question is set in the questionnaire asking about the overall satisfaction level of the class, and the object of the CS analysis is to examine how the score can be raised. Other questions that influence the satisfaction of the class are set. For example, as shown in Table I, Q 11 asks about the "overall satisfaction of the class," and Q 01 to Q 10 are questions that influence Q 11. The items are scored on a five-point scale: 5-Strongly Agree, 4-Agree, 3-Neither Agree nor Disagree, 2-Disagree, 1-Strongly Disagree. The CS analysis is performed using the weighted mean of the score of the five-point scale responses, with Q 11 as the response variable and $Q 01$ to $Q 10$ as the explanatory variables.

Table I

Questionnaire Items

\begin{tabular}{|c|c|c|}
\hline Number & Question Statement & Handling of Variables \\
\hline Q 01 & The students were able to acquire the knowledge, skills, etc., listed in the "Attainment Targets" of the syllabus. & Explanatory variable \\
\hline Q 02 & The class was conducted according to the "Class Format and Plan" in the syllabus. & Explanatory variable \\
\hline Q 03 & The difficulty level of the class was appropriate for me. & Explanatory variable \\
\hline Q 04 & The classes have increased my interest in related subjects and fields. & Explanatory variable \\
\hline Q 05 & The class was conducted in an environment and atmosphere conducive to learning. & Explanatory variable \\
\hline Q 06 & Care was taken to ensure that the class content was well understood. & Explanatory variable \\
\hline Q 07 & Preparations for the use of teaching materials and equipment were well done. & Explanatory variable \\
\hline Q 08 & The instructor's speech, language, and attitude were appropriate. & Explanatory variable \\
\hline Q 09 & The instructor's course instruction reflected the students' degree of understanding and their academic level. & Explanatory variable \\
\hline Q 10 & Guidance on learning outside the classroom (how to research literature, etc.) was appropriate. & Explanatory variable \\
\hline Q 11 & Overall, this course was beneficial. & Response variable \\
\hline
\end{tabular}

Rating scale: 5-Strongly Agree, 4-Agree, 3- Neither Agree nor Disagree, 2-Disagree, 1-Strongly Disagree.

After administering the course evaluation questionnaire, the weighted mean values of each question are calculated in the aggregated results (Table II). Next, the correlation coefficients between the question on overall satisfaction (Q 11) and each of the questions from Q 01 to Q 10 (Table III) are calculated. These data are based on simulated data since the questionnaire is based on a five-point scale ranging from "5-Strongly Agree" to "1-Strongly Disagree"; therefore, the weighted mean value of the responses is between 1.0 and 5.0 (Table II). Since the possible range of values for the correlation coefficient is -1.0 to 1.0 , it can be negative in Table III. However, a strong negative correlation is rarely observed because Q 01 to $Q 10$ are questions that would contribute to overall class satisfaction (Q 11). 
Table II

Weighted Mean of each Response

\begin{tabular}{ll}
\hline Number & Weighted Mean Value of each Respondent \\
\hline Q 01 & 4.0 \\
Q 02 & 3.8 \\
Q 03 & 2.7 \\
Q 04 & 2.5 \\
Q 05 & 3.4 \\
Q 06 & 4.3 \\
Q 07 & 4.1 \\
Q 08 & 3.2 \\
Q 09 & 3.5 \\
Q 10 & 3.1 \\
Q 11 & $\mathbf{3 . 6}$ \\
\hline
\end{tabular}

Table III

The Correlation Coefficient between Q 11 and Q 01 to Q 10

\begin{tabular}{ll}
\hline Number & Correlation Coefficients \\
\hline Q 01 & 0.17 \\
Q 02 & 0.51 \\
Q 03 & 0.39 \\
Q 04 & 0.62 \\
Q 05 & 0.39 \\
Q 06 & 0.38 \\
Q 07 & 0.59 \\
Q 08 & 0.61 \\
Q 09 & -0.12 \\
Q 10 & 0.12 \\
\hline
\end{tabular}

Next, Q 01 to Q 10 are plotted on the graph, with the correlation coefficients on the horizontal axis and the weighted mean of the responses on the vertical axis, as shown in Fig. 1. The weighted mean of the responses on the vertical axis shows higher satisfaction at the top and lower satisfaction at the bottom. For the correlation coefficient on the horizontal axis, the right side had a higher correlation with the overall satisfaction of Q11. Therefore, if the question items plotted on the lower right ( $Q 04$ in this case) are improved, the overall satisfaction level of Q 11 can be increased. The results indicate that the class should be improved concerning Q 04, and if the content of the class is improved to raise the rating in Q 04, the overall satisfaction level will be improved. In other words, the results of the CS analysis will lead to improvements in teaching.

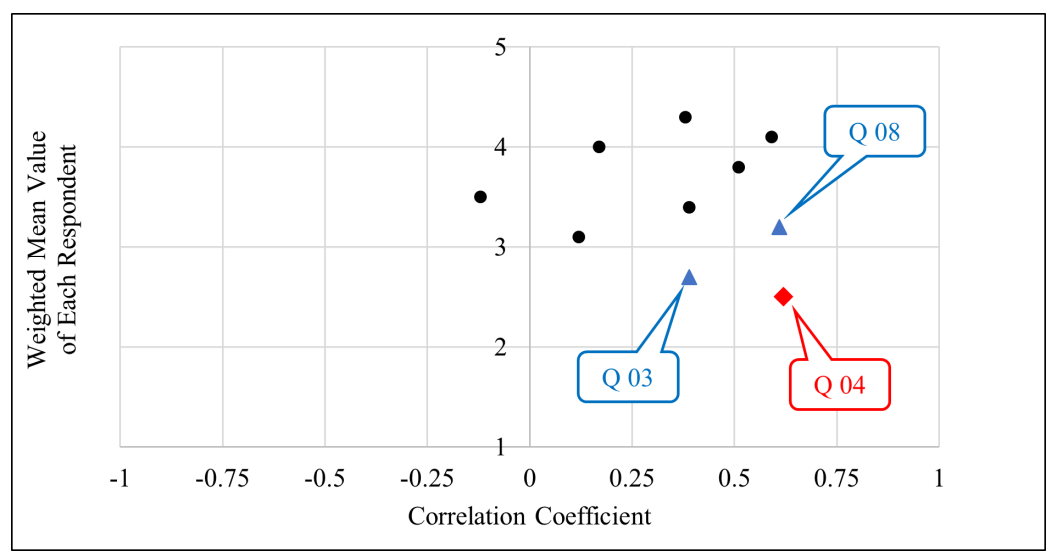

Fig. 1. CS graph

From here, what the next priority items for improvement should be after improving $Q 04$ is considered. It is suggested that $Q 03$ or $Q 08$ should be improved since the lower and the right questions in Fig. 1 are the more important items. Previous studies have shown quantitatively evaluating methods (Dohi et al., 2012; Matsumoto \& Tsukamoto, 2004; Minami, 2007). For the quantitative evaluation, firstly, a CS portfolio (Hamada et al., 2013) is created by converting the values of the vertical and horizontal axes into deviation values. The CS portfolio is classified into Priority Maintenance Areas, Maintenance Areas, Improvement Areas, and Priority Improvement Areas (Gök, 2009). Secondly, an index called the Improvement Level Index (ILI) is calculated using the CS portfolio. Finally, the ILI is used to quantitatively evaluate which question needs to be improved. Although these methods are well established in terms of research, they are complicated and time-consuming; therefore, from the perspective of IRTL, it is difficult to say that they are practical.

As a result, in order to improve class content by utilizing the course evaluation questionnaires, it is suggested that each higher education institution provide each instructor with feedback that improves the items plotted in the lower right of the CS graph shown in Fig. 1. From the perspective of IRTL, this is the practically feasible analysis outline of course evaluation questionnaires for each higher education institution. 


\section{Discussion}

In the previous section, the analysis method of the course evaluation questionnaire using the CS analysis from the perspective of IRTL is described. This section discusses whether this CS analysis can be applied to other questionnaires in higher education institutions.

In Japan, due to the expansion of IR activities in recent years, each higher education institution has been conducting its original student surveys in various forms (The University of Tokyo, n.d.; Waseda University, 2020). However, there are diverse survey objectives and methods. It is difficult for each higher education institution to use the survey results for appropriate benchmarking and effective improvement of educational content (Ministry of Education, Culture, Sports, Science and Technology (MEXT), n.d.). In Japan, the National Institute for Educational Policy Research (NIER) conducted a nationwide survey on learning conditions, but the survey was conducted randomly (National Institute for Educational Policy Research (NIER), 2016). In other words, although each higher education institution has conducted surveys, no survey of all students in Japan has been conducted, and data on nationwide student surveys have not been maintained and utilized (Ministry of Education, Culture, Sports, Science and Technology (MEXT), n.d.). In Europe and the United States, large-scale student surveys, such as the National Student Survey in the United Kingdom, the National Survey of Student Engagement, and the Cooperative Institutional Research Program in the United States, are conducted to understand the status of student learning. The obtained data are generally used as evidence data for accreditation and improvement of educational content (Ministry of Education, Culture, Sports, Science and Technology (MEXT), n.d.). Therefore, in Japan, the MEXT and NIER jointly conducted a large-scale nationwide student survey on a trial basis in the academic year 2019 with the aim of organizing and verifying survey methods and questions to be used for various purposes, such as improving university education and planning national policies. The findings of this survey will be used for various purposes, such as improving university education and planning national policies. The survey results are published (Ministry of Education, Culture, Sports, Science and Technology (MEXT), 2020b) and the percentages of responses are tabulated by the establishment (national/public/private), faculty size, and faculty ield. While the data obtained are useful for under-standing the actual conditions of students, it is difficult to say whether the data can be analyzed at a level that will be useful for improving educational content. Although, the nation-wide student survey conducted in the academic year 2019 was in the trial stage, it is expected that the second nationwide student survey to be conducted in the academic year 2021 will include an analysis at the national level that will enable the beneficial application of the data obtained.

Suppose a nationwide student survey is conducted jointly by MEXT and NIER. In that case, sufficient resources can be invested to collect data on a nationwide basis and conduct various statistical analyses. It will be possible to obtain effective analytical results to understand students' actual situation and improve educational content. Incidentally, student surveys of each higher education institution will continue to be conducted even if a nationwide student survey is conducted. In this case, except for higher education institutions where IRTL organizations are fully functional, it is difficult to obtain useful analysis results to fulfill the IRTL's goal of improving education and learning and the course evaluation questionnaire.

Additionally, there could be duplicate questions in the questionnaires administered in the student surveys at each university and in the National Student Survey. Therefore, questionnaire-based surveys should not end merely by tabulating the percentage of responses to the questionnaire results. Rather, they should be conducted in a manner that improves teaching and learning and leads to quality assurance of education by carefully considering "how to analyze the results and what kind of findings should be obtained" in advance, for example, as in the previously discussed method using CS analysis. Otherwise, it will be a gross wastage of a huge amount of data collected at great expense and time.

\section{Limitations and Future Research Directions}

This study proposed a simplified CS analysis procedure, which can be practically applied to questionnaire analysis from the perspective of IRTL. However, this method proposed in this paper has not been used by higher education institutions. In future, each higher education institutions should analyze the results of the questionnaires by the method and scholars are encouraged to verify the usefulness of this method.

\section{Conclusion}

In analyzing the questionnaire surveys conducted in higher education institutions, merely tabulating the percentage of responses does not lead to appropriate teaching and learning improvement and educational quality assurance. Therefore, methods using CS analysis and text mining have been investigated to solve this problem. However, although these methods have been established for research, they are difficult to understand, thus not being feasible for large-scale implementation until the IRTL organization is fully operational. Furthermore, even if practically possible, the analysis results cannot be used effectively unless higher education institutions are active in FD activities. Therefore, the simple CS analysis proposed in this study or, for example, cross-tabulation, can be practically implemented by the IRTL organization of each higher education institution. In addition, each institution must select and implement suitable methods that will enable faculty members not familiar with statistical methods to utilize the analysis results effectively following appropriate FD activities. The next challenge will be for education experts to disseminate these specific methods to educational institutions through case studies.

\section{References}

Abe, T., Tajima, T., \& Kimura, H. (2006). Analysis of questionnaire using multivariate analysis for improving lectures. Journal of JSEE, 54(6), 136-140. doi:https://doi.org/10.4307/jsee.54.6_136

Dohi, S., Miyakawa, O., \& Konno, N. (2012). Continuous CS analysis of using the SIEM to introduction to computer programming education in the school of engineering evening division at the department of electrical and electronic engineering. Journal of JSEE, 60(4), 56-62. doi:https://doi.org/10.4307/jsee.60.4_56

Endo, T. (2008). Gakusei ni yoru jugyō hyōka no CS bunseki [CS analysis of course evaluation by students] (in Japanese). Saga Journal of Higher Education, 4,1-10. 
Fushikida, W., Kitamura, S., \& Yamauchi, Y. (2012). Analysis of appeal and dissatisfaction in seminars for third- and fourth-year students with text mining. Japan Journal of Educational Technology, 36(Suppl.), 165-168. doi:https://doi.org/10.15077/jjet.KJ00008609803

Gök, O. (2009). Linking account portfolio management to customer information: Using customer satisfaction metrics for portfolio analysis. Industrial Marketing Management, 38(4), 433-439. doi:https://doi.org/10.1016/j.indmarman.2008.02.010

Hamada, H., Hashimoto, T., Ishizuka, R., Nishida, N., Nishikawa, A., \& Nakagawa, N. (2013). Anxiety factors of students immediately before practical care training: CS portfolio analysis. Rigakuryoho Kagaku, 28(1), 39-43. doi:https://doi.org/10.1589/rika.28.39

Kamaga, S. (2015). Analysis and measures to improve comments section of class evaluations using text mining. Shokei Educational Institution Bulletin, 47, 49-61. doi:https://doi.org/10.24577/seia.47.0_49

Kawakami, M., \& Yoshizumi, K. (2008). Characterization of questionnaires of college student evaluation on lectures with respect to faculty development. Educational Information Research, 24(2), 57-67. doi:https://doi.org/10.20694/jjsei.24.2_57

Kurita, K. (2006). On-line course evaluation in the U.S.: A case study. Research on Academic Degrees and University Evaluation, 4, 105-113.

Matsuda, T., \& Watanabe, Y. (2017). IR for educational planning, learning analytics, and educational engineering. Japan Journal of Educational Technology, 41(3), 199-208. doi:https://doi.org/10.15077/jjet.42028

Matsumoto, Y., \& Tsukamoto, Y. (2004). Analysis of lecture evaluations and point quantification for teaching improvements based on the concept of customer satisfaction analysis. Kyoto University Researches in Higher Education, 10, 21-32.

Minami, M. (2007). Gakusei ni yoru jugyō hyōka e no CS bunseki no tekiyo" [Application of CS analysis to courses evaluation by students] (in Japanese). Bulletin of the Integrated Center for Educational Research and Practice, Mie University, 27, 29-34.

Minami, M. (2008). A study of indices of need to improvement based on the customer satisfaction analyses on class evaluations. Bulletin of the Faculty of Education, Mie University, 59, 291-297.

Minami, M., \& Nakanishi, Y. (2010). Evaluation and improvement of couses using CS analysis. Bulletin of the Integrated Center for Educational Research and Practice, 30, 39-43.

Ministry of Education, Culture, Sports, Science and Technology (MEXT). (n.d.). Zenkoku gakusei chōsa [Nationwide student survey] (in Japanese). Retrieved from https://bit.1y/350Vdd5

Ministry of Education, Culture, Sports, Science and Technology (MEXT). (2014). Daigaku ni okeru kyōiku naiyō-tō no kaikaku jōkyō ni tsuite (gaiyō) [Reform status of educational content, etc. at universities (summary)] (in Japanese). Retrieved from https://bit. ly/2UrgR4C

Ministry of Education, Culture, Sports, Science and Technology (MEXT). (2019). Reiwa gan'nendo 'zenkoku gakusei chōsa (shikō jisshi)' ni tsuite [About "nationwide student survey (trial implementation)" in the academic year 2019] (in Japanese). Retrieved from https://bit.1y/3dbnsH1

Ministry of Education, Culture, Sports, Science and Technology (MEXT). (2020a). Reiwa gan'nendo 'zenkoku gakusei chōsa (shikō jisshi)' kekka” [Result of "nationwide student survey (trial implementation) in the academic year 2019] (in Japanese). Retrieved from https://bit.1y/3i6SQJt

Ministry of Education, Culture, Sports, Science and Technology (MEXT). (2020b). Reform status of educational content, etc. at universities in the academic year 2018. Retrieved from https://bit.1y/3qmEPdn

National Institute for Educational Policy Research (NIER). (2016). Daigakusei no gakushū jittai ni kansuru chōsa kenkyū ni tsuite (gaiyō) [Survey research on the actual learning situation of university students (summary)] (in Japanese). Retrieved from https://bit.ly/3j83JM3

Okada, Y., \& Torii, T. (2018). Process model of educational information management in institutional research for teaching and learning. Japan Journal of Educational Technology, 42, 313-322. doi:https://doi.org/10.15077/jjet.42109

Takahashi, K., Hayashi, Y., Taneda, Y., Kagei, S., Yaguchi, T., \& Kanzaki, N. (2005). School-wide course improvement and course evaluations: Report on current status of program. Kyoto University Researches in Higher Education, 11, 19-32.

The University of Chicago. (n.d.). Course evaluations. Retrieved from https://bit.1y/3j5YSv0

The University of Tokyo. (n.d.). Daigaku kyōiku no tassei-do chōsa [Survey on achievement in university education] (in Japanese). Retrieved from https : // bit.ly/3i8rMd9

Tohoku University. (n.d.). Zengaku onrain jugyō ankēto' no kekka gaiyō [Summary of the results of the "Tohoku university online course questionnaire"] (in Japanese). Retrieved from https://bit.1y/3x7fovs

Waseda University. (2020). Onrain jugyō ni kansuru chōsa kekka [Survey results on online courses] (in Japanese). Retrieved from https ://bit . ly/3j85nxd

Yamada, R. (2016). The development process of IR in the United States: Analysis of the function of IR in the framework of higher education policy and the issue of professionalism. Japanese Journal of Higher Education Research, 19, 25-47. doi:https://doi.org/10.32116/jaher.19.0_25 